\title{
Renormalization of B-meson distribution amplitudes
}

\section{N. Offen*}

Laboratoire de Physique Théorique

CNRS/Univ. Paris-Sud 11 (UMR 8627),

F-91405 Orsay, France

E-mail: nils.offeneth.u-psud.fr

\section{S. Descotes-Genon}

Laboratoire de Physique Théorique

CNRS/Univ. Paris-Sud 11 (UMR 8627),

F-91405 Orsay, France

E-mail: sebastien.descotes-genon@th.u-psud.fr

\begin{abstract}
We summarize a recent calculation of the evolution kernels of the two-particle $B$-meson distribution amplitudes $\phi_{+}$and $\phi_{-}$taking into account three-particle contributions. In addition to a few phenomenological comments, we give as a new result the evolution kernel of the combination of three-particle distribution amplitudes $\Psi_{A}-\Psi_{V}$ and confirm constraints on $\phi_{+}$and $\phi_{-}$derived from the light-quark equation of motion.
\end{abstract}

International Workshop on Effective Field Theories: from the pion to the upsilon February 2-6 2009

Valencia, Spain

\footnotetext{
* Speaker.
} 


\section{Introduction}

Exclusive decays of $B$-mesons provide important tools to test the Standard Model and to search for physics beyond it. Hadronic inputs encoding soft physics are not only form factors but also light-cone distribution amplitudes (LCDAs). In particular the $B$-meson LCDAs enter the parametrization of the hard-scattering part of hadronic matrix elements of bilocal current operators where large momentum is tranferred to the soft spectator quark [1-10]. Impressive progress has been made in the calculation of the hard scattering amplitudes entering factorization theorems, see e.g. [11-15] for the $B \rightarrow P P$ case, but one limiting factor for the extraction of fundamental parameters is the uncertainty coming from the hadronic input. Recent years have seen several analyses concerning the renormalization properties $[16,17,18]$ and the shape of the $B$-meson LCDAs $[4,18,19,20,21,22,23]$. Up to now these analyses were restricted to the two-particle case or to leading order with the exception of [23]. Here we present the results of [24] for the renormalization of the two-particle $B$-meson LCDAs taking into account mixing with three-parton LCDAs and in section (2.3) the results of a new calculation for the combination of three-particle LCDAs $\Psi_{A}-\Psi_{V}$ entering the equations of motion.

\section{One-loop calculation with three-parton external state}

The relevant two- and three-parton distribution amplitudes are defined as $B$ to vacuum matrixelements of a non-local heavy-to-light operator, which reads in the two-particle case [4]

$$
\left\langle 0\left|\bar{q}_{\beta}(z)[z, 0]\left(h_{v}\right)_{\alpha}(0)\right| B(p)\right\rangle=-i \frac{\hat{f}_{B}(\mu)}{4}\left[(1+v)\left(\tilde{\phi}_{+}(t)+\frac{t}{2 t}\left[\tilde{\phi}_{-}(t)-\tilde{\phi}_{+}(t)\right]\right) \gamma_{5}\right]_{\alpha \beta}
$$

and in the three-particle case [21] (the most general decomposition without contraction with a light-like vector is given in [25]):

$$
\begin{gathered}
\left\langle 0\left|\bar{q}_{\beta}(z)[z, u z] g G_{\mu v}(u z) z^{v}[u z, 0]\left(h_{v}\right)_{\alpha}(0)\right| B(p)\right\rangle \\
=\frac{\hat{f}_{B}(\mu) M}{4}\left[( 1 + v ) \left[\left(v_{\mu} t-t \gamma_{\mu}\right)\left(\tilde{\Psi}_{A}(t, u)-\tilde{\Psi}_{V}(t, u)\right)-i \sigma_{\mu v} z^{v} \tilde{\Psi}_{V}(t, u)\right.\right. \\
\left.\left.\quad-z_{\mu} \tilde{X}_{A}(t, u)+\frac{z_{\mu} z}{t} \tilde{Y}_{A}(t, u)\right] \gamma_{5}\right]_{\alpha \beta} .
\end{gathered}
$$

We use light-like vectors $n_{ \pm}$so that every vector can be decomposed as

$$
\begin{aligned}
& q_{\mu}=\left(n_{+} \cdot q\right) \frac{n_{-, \mu}}{2}+\left(n_{-} \cdot q\right) \frac{n_{+, \mu}}{2}+q_{\perp \mu}=q_{+} \frac{n_{-, \mu}}{2}+q_{-} \frac{n_{+, \mu}}{2}+q_{\perp \mu}, \\
& n_{+}^{2}=n_{-}^{2}=0 \quad n_{+} \cdot n_{-}=2 \quad v=\left(n_{+}+n_{-}\right) / 2 .
\end{aligned}
$$

The computation of the renormalisation properties of the distribution amplitudes requires us to consider matrix elements of the relevant operators

$$
\begin{aligned}
O_{+}^{H}(\omega) & =\frac{1}{2 \pi} \int d t e^{i \omega t}\left\langle 0\left|\bar{q}(z)[z, 0] \boldsymbol{n}_{+} \Gamma h_{v}(0)\right| H\right\rangle \\
O_{-}^{H}(\omega) & =\frac{1}{2 \pi} \int d t e^{i \omega t}\left\langle 0\left|\bar{q}(z)[z, 0] \boldsymbol{n}_{-} \Gamma h_{v}(0)\right| H\right\rangle \\
O_{3}^{H}(\omega, \xi) & =\frac{1}{(2 \pi)^{2}} \int d t e^{i \omega t} \int d u e^{i \xi u t}\left\langle 0\left|\bar{q}(z)[z, u z] g_{s} G_{\mu v}(u z) z^{v}[u z, 0] \Gamma h_{v}(0)\right| H\right\rangle
\end{aligned}
$$




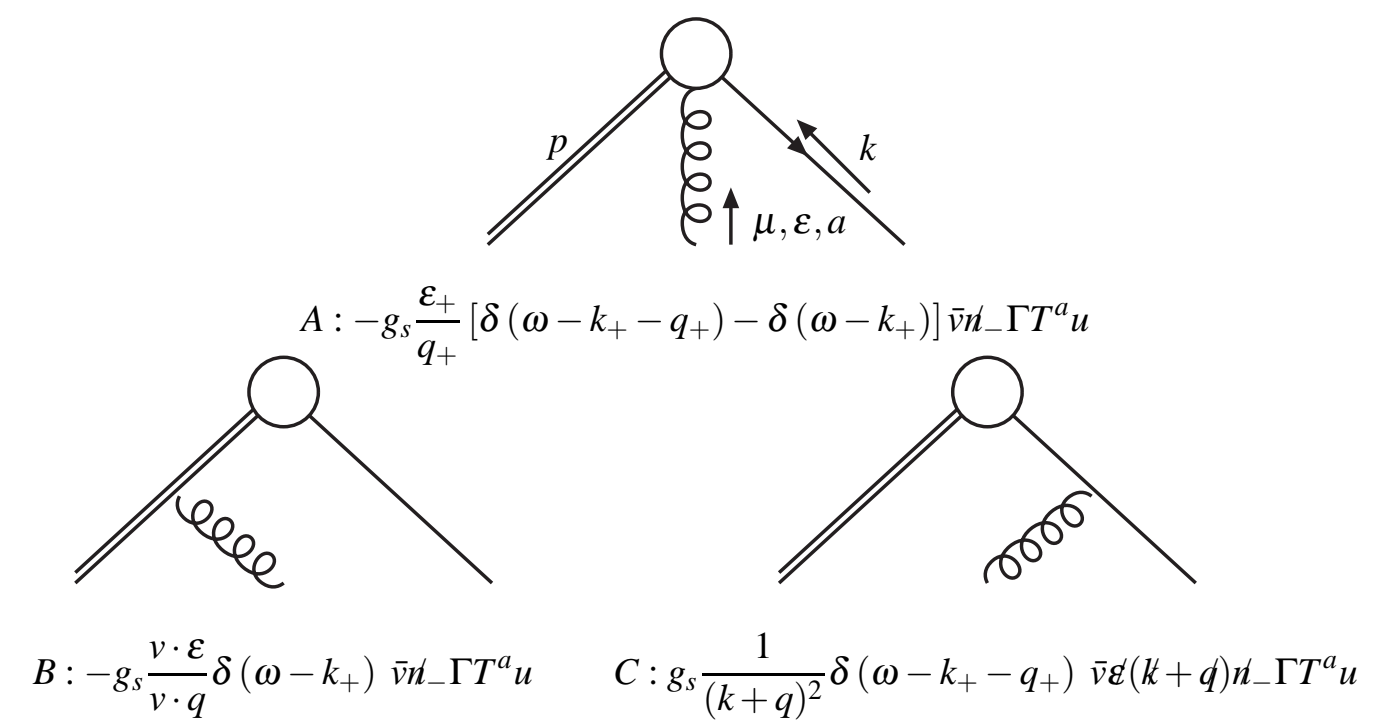

Figure 1: The three leading-order contributions to the matrix element of $O_{ \pm}$with a three-parton external state.

with $z$ parallel to $n_{+}$, i.e. $z_{\mu}=t n_{+, \mu}, t=v \cdot z=z_{-} / 2$ and the path-ordered exponential in the $n_{+}$ direction:

$$
\begin{aligned}
{[z, 0] } & =P \exp \left[i g_{s} \int_{0}^{z} d y_{\mu} A^{\mu}(y)\right] \\
& =1+i g_{s} \int_{0}^{1} d \alpha z_{\mu} A^{\mu}(\alpha z)-g_{s}^{2} \int_{0}^{1} d \alpha \int_{0}^{\alpha} d \beta z_{\mu} z_{v} A^{\mu}(\alpha z) A^{v}(\beta z)+\ldots
\end{aligned}
$$

The Fourier-transforms of the different distribution amplitudes are then defined via

$$
\phi_{ \pm}(\omega)=\frac{1}{2 \pi} \int d t e^{i \omega t} \tilde{\phi}_{ \pm}(t) \quad F(\omega, \xi)=\frac{1}{(2 \pi)^{2}} \int d t \int d u t e^{i(\omega+u \xi) t} \tilde{F}(t, u)
$$

where $F=\Psi_{V}, \Psi_{A}, X_{A}, Y_{A}$. Since the renormalization of the operators is independent of the infrared properties of the matrix-elements, we can choose an on-shell partonic external state consisting of a light quark, a heavy quark and a gluon in equation (2.6). The resulting leading-order diagrams are shown in figures 1 and 2. Next-to-leading order (NLO) diagrams are obtained by adding a gluon or a quark loop (a ghost loop) in all possible places (for a complete list of diagrams, see [24]). Since the operators give rise to $\delta$-distributions in the +-component of the momenta, we chose to proceed via the theorem of residues. To be more explicit, we decomposed the loop momentum $l$ in light-cone components, picked up the poles in the $l_{-}$-integral and performed the $l_{\perp}$-integration in dimensional regularization with $D=2-2 \varepsilon$ dimensions. Additional $\frac{1}{\varepsilon}$-poles arise through the $l_{+}$-integration for diagrams where a gluon is exchanged between the Wilson-line from the operator and the heavy-quark field. These are related to the cusp anomalous dimension, see e.g [26, 27], stemming from the intersection of one light-like Wilson line from the path ordered exponential in the operator and one time-like Wilson line from the interaction of soft gluons with the heavy quark. The additional poles give rise to $\frac{1}{\varepsilon^{2}}$-terms as well as Sudakov logarithms. 


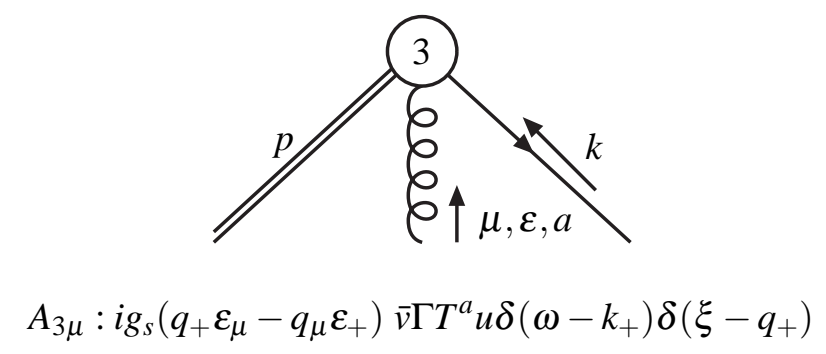

Figure 2: Leading-order contribution to the matrix element of $O_{3 \mu}$ with a three-parton external state.

\section{$2.1 \phi_{+}$-case}

In the $\phi_{+}$-case there is no mixing from three-particle distribution amplitudes. $\gamma_{+, 3}=0$ at order $\alpha_{s}$. We confirm the result for the anomalous-dimension matrix found in [16]

$$
\gamma_{+}^{(1)}\left(\omega, \omega^{\prime} ; \mu\right)=\left(\Gamma_{\text {cusp }}^{(1)} \log \frac{\mu}{\omega}+\gamma^{(1)}\right) \delta\left(\omega-\omega^{\prime}\right)-\Gamma_{\text {cusp }}^{(1)} \omega\left(\frac{\theta\left(\omega^{\prime}-\omega\right)}{\omega^{\prime}\left(\omega^{\prime}-\omega\right)}+\frac{\theta\left(\omega-\omega^{\prime}\right)}{\omega\left(\omega-\omega^{\prime}\right)}\right)_{+}
$$

with

$$
\left[f\left(\omega, \omega^{\prime}\right)\right]_{+}=f\left(\omega, \omega^{\prime}\right)-\delta\left(\omega-\omega^{\prime}\right) \int d \omega^{\prime} f\left(\omega, \omega^{\prime}\right) \quad \Gamma_{\text {cusp }}^{(1)}=4 \quad \gamma^{(1)}=-2
$$

\section{$2.2 \phi_{-}$-case}

The $\phi_{-}$case is more involved. After coupling-constant and external leg renormalization there remains a genuine three-particle term. The renormalization group equation to order $\alpha_{s}$ can be written as

$$
\begin{aligned}
\frac{\partial \phi_{-}(\omega ; \mu)}{\partial \log \mu}= & -\frac{\alpha_{s}(\mu)}{4 \pi}\left(\int d \omega^{\prime} \gamma_{-}^{(1)}\left(\omega, \omega^{\prime} ; \mu\right) \phi_{-}\left(\omega^{\prime} ; \mu\right)\right. \\
& \left.+\int d \omega^{\prime} d \xi^{\prime} \gamma_{-, 3}^{(1)}\left(\omega, \omega^{\prime}, \xi^{\prime} ; \mu\right)\left[\Psi_{A}-\Psi_{V}\right]\left(\omega^{\prime}, \xi^{\prime} ; \mu\right)\right)
\end{aligned}
$$

where $\gamma_{-}^{(1)}$ is the result from [17]

$$
\gamma_{-}^{(1)}\left(\omega, \omega^{\prime} ; \mu\right)=\gamma_{+}^{(1)}-\Gamma_{\text {cusp }}^{(1)} \frac{\theta\left(\omega^{\prime}-\omega\right)}{\omega^{\prime}}
$$

and $\gamma_{-, 3}^{(1)}$ from [24]

$$
\begin{array}{r}
\gamma_{-, 3}^{(1)}\left(\omega, \omega^{\prime}, \xi^{\prime}\right)=4\left[\frac { \Theta ( \omega ) } { \omega ^ { \prime } } \left\{\left(C_{A}-2 C_{F}\right)\left[\frac{1}{\xi^{\prime 2}} \frac{\omega-\xi^{\prime}}{\omega^{\prime}+\xi^{\prime}-\omega} \Theta\left(\xi^{\prime}-\omega\right)+\frac{\Theta\left(\omega^{\prime}+\xi^{\prime}-\omega\right)}{\left(\omega^{\prime}+\xi^{\prime}\right)^{2}}\right]\right.\right. \\
\left.\left.-C_{A}\left[\frac{\Theta\left(\omega^{\prime}+\xi^{\prime}-\omega\right)}{\left(\omega^{\prime}+\xi^{\prime}\right)^{2}}-\frac{1}{\xi^{\prime 2}}\left(\Theta\left(\omega-\omega^{\prime}\right)-\Theta\left(\omega-\omega^{\prime}-\xi^{\prime}\right)\right)\right]\right\}\right]_{+}
\end{array}
$$

where we defined the + -distribution with three variables as

$$
\left[f\left(\omega, \omega^{\prime}, \xi^{\prime}\right)\right]_{+}=f\left(\omega, \omega^{\prime}, \xi^{\prime}\right)-\delta\left(\omega-\omega^{\prime}-\xi^{\prime}\right) \int d \omega f\left(\omega, \omega^{\prime}, \xi^{\prime \prime}\right)
$$




\section{3 $\Psi_{A}-\Psi_{V}$-renormalization and equation of motion constraints}

Here we report on an up to now unpublished calculation of the renormalization of the threeparticle LCDAs $\Psi_{A}-\Psi_{V}$. We project on the relevant distribution amplitudes in equation (2.3) using $\Gamma=\gamma_{\perp}^{\mu} \boldsymbol{h}_{+} \boldsymbol{h}_{-} \gamma_{5}$ (although a $\gamma^{\mu}$ instead of $\gamma_{\perp}^{\mu}$ yields the same result). The calculations go along the same lines as in the previous two cases, even though there is only one leading-order structure (shown in figure 2) and NLO diagrams must have one gluon attached to the vertex in order not to vanish trivially. For convenience the result is splitted into $C_{F}$ - and $C_{A}$-colour structures.

$$
\begin{aligned}
\gamma_{3,3, C_{A}}^{(1)}\left(\omega, \xi, \omega^{\prime}, \xi^{\prime}\right) & =2\left[\delta\left(\omega-\omega^{\prime}\right)\left\{\frac{\xi}{\xi^{\prime 2}} \Theta\left(\xi^{\prime}-\xi\right)-\left[\frac{\Theta\left(\xi-\xi^{\prime}\right)}{\xi-\xi^{\prime}}\right]_{+}-\left[\frac{\xi}{\xi^{\prime}} \frac{\Theta\left(\xi^{\prime}-\xi\right)}{\xi^{\prime}-\xi}\right]_{+}\right\}\right. \\
& +\delta\left(\xi-\xi^{\prime}\right)\left\{\left[\frac{\Theta\left(\omega-\omega^{\prime}\right)}{\omega-\omega^{\prime}}\right]_{+}+\left[\frac{\omega}{\omega^{\prime}} \frac{\Theta\left(\omega^{\prime}-\omega\right)}{\omega^{\prime}-\omega}\right]_{+}\right\}+\delta\left(\omega+\xi-\omega^{\prime}-\xi^{\prime}\right) \\
& \times\left\{\frac{1}{\xi^{\prime}} \Theta\left(\omega-\omega^{\prime}\right)-\left[\frac{\Theta\left(\omega-\omega^{\prime}\right)}{\omega-\omega^{\prime}}\right]_{+}-\left[\frac{\omega}{\omega^{\prime}} \frac{\Theta\left(\omega^{\prime}-\omega\right)}{\omega^{\prime}-\omega}\right]_{+}\right\} \\
& +\delta\left(\omega+\xi-\omega^{\prime}-\xi^{\prime}\right) \frac{1}{\xi^{\prime}\left(\omega^{\prime}+\xi^{\prime}\right)}\left\{\frac{\omega-\xi^{\prime}}{\xi^{\prime}}\left(\omega^{\prime}+\xi^{\prime}-\omega\right) \Theta\left(\omega-\omega^{\prime}\right)\right. \\
& -\frac{\omega}{\omega^{\prime}}\left(\omega^{\prime}+2 \xi^{\prime}-\omega\right) \Theta\left(\omega^{\prime}-\omega\right) \Theta(\omega)+\frac{\omega}{\xi^{\prime}}\left(\omega-\xi^{\prime}\right) \Theta\left(\xi^{\prime}-\omega\right) \Theta(\omega) \\
& \left.\left.+\frac{\omega-\xi^{\prime}}{\omega^{\prime}}\left(\omega^{\prime}+\xi^{\prime}-\omega\right) \Theta\left(\omega-\xi^{\prime}\right) \Theta(\xi)\right\}\right] \\
\gamma_{3,3, C_{F}}^{(1)}\left(\omega, \xi, \omega^{\prime}, \xi^{\prime} ; \mu\right) & =\gamma_{+}^{(1)}\left(\omega, \omega^{\prime} ; \mu\right) \delta\left(\xi-\xi^{\prime}\right)+\gamma_{R 3,3}^{(1)}\left(\omega, \xi, \omega^{\prime}, \xi^{\prime}\right) \\
\gamma_{R 3,3}^{(1)}\left(\omega, \xi, \omega^{\prime}, \xi^{\prime}\right) & =4 \delta\left(\omega+\xi-\omega^{\prime}-\xi^{\prime}\right) \\
& \times\left[\frac{\xi^{2}}{\omega^{\prime}} \frac{\Theta\left(\omega^{\prime}-\xi\right)}{(\omega+\xi)^{2}} \Theta(\xi)+\frac{\omega}{\xi^{\prime}} \frac{\Theta\left(\xi-\omega^{\prime}\right)}{\omega+\xi} \Theta(\omega)\left(\frac{\xi}{\omega+\xi}-\frac{\omega-\xi^{\prime}}{\xi^{\prime}}\right)\right]
\end{aligned}
$$

with $\gamma_{+}^{(1)}$ the same as in equation (2.10) and $\gamma_{3,3}^{(1)}$ defined as in (2.11) with obvious changes. Part of this calculation, namely the light-quark-gluon part, has been calculated in a different context and a different scheme, e.g. in [29, 28].

In [21] two equations from the light- and heavy-quark equations of motion were derived

$$
\omega \phi_{-}^{\prime}(\omega ; \mu)+\phi_{+}(\omega ; \mu)=I(\omega ; \mu), \quad(\omega-2 \bar{\Lambda}) \phi_{+}(\omega ; \mu)+\omega \phi_{-}(\omega ; \mu)=J(\omega ; \mu),
$$

where $I(J)(\omega ; \mu)$ are integro-differential expressions involving the three-particle LCDAs $\Psi_{A}-\Psi_{V}$ $\left(\Psi_{A}+X_{A}\right.$ and $\left.\Psi_{V}\right)$ respectively. While the second equation was shown not to hold beyond leading order in $[17,23]$ we have checked that the first one is valid once renormalization is taken into account. Taking the derivative of the first equation with respect to $\log \mu$, inserting

$$
I(\omega ; \mu)=2 \frac{d}{d \omega} \int_{0}^{\omega} d \rho \int_{\omega-\rho}^{\infty} \frac{d \xi}{\xi} \frac{\partial}{\partial \xi}\left[\Psi_{A}(\rho, \xi ; \mu)-\Psi_{V}(\rho, \xi ; \mu)\right]
$$

and using the relation from [17]

$$
-\omega \frac{d}{d \omega} \int_{0}^{\eta} \frac{d \omega^{\prime}}{\eta} \gamma_{-}^{(1)}\left(\omega, \omega^{\prime} ; \mu\right)=\gamma_{+}^{(1)}(\omega, \eta ; \mu)
$$


one arrives at the following equation

$$
\begin{aligned}
& \omega \frac{d}{d \omega} \int d \omega^{\prime} d \xi^{\prime} \gamma_{-, 3}^{(1)}\left(\omega, \omega^{\prime}, \xi^{\prime}\right)\left(\Psi_{A}\left(\omega^{\prime}, \xi^{\prime} ; \mu\right)-\Psi_{V}\left(\omega^{\prime}, \xi^{\prime} ; \mu\right)\right) \\
+ & 2 \int d \omega^{\prime} \gamma_{+}^{(1)}\left(\omega, \omega^{\prime} ; \mu\right) \frac{d}{d \omega^{\prime}} \int_{0}^{\omega^{\prime}} d \rho \int_{\omega^{\prime}-\rho}^{\infty} \frac{d \xi}{\xi} \frac{\partial}{\partial \xi}\left(\Psi_{A}(\rho, \xi ; \mu)-\Psi_{V}(\rho, \xi ; \mu)\right) \\
= & 2 \int d \omega^{\prime} d \xi^{\prime} \frac{d}{d \omega} \int_{0}^{\omega} d \rho \int_{\omega-\rho}^{\infty} \frac{d \xi}{\xi} \frac{\partial}{\partial \xi} \gamma_{3,3}^{(1)}\left(\rho, \xi, \omega^{\prime} \xi^{\prime} ; \mu\right)\left(\Psi_{A}\left(\omega^{\prime}, \xi^{\prime} ; \mu\right)-\Psi_{V}\left(\omega^{\prime}, \xi^{\prime} ; \mu\right)\right),
\end{aligned}
$$

which can be proven to hold at order $\alpha_{s}$ by simple insertion of the respective evolution kernels (2.10), (2.13), (2.15), (2.16). This non-trivial outcome gives us further confidence concerning the renormalization group properties of the LCDAs.

\section{Conclusions}

The presence of $\delta\left(\omega-\omega^{\prime}\right) \log (\mu / \omega)$ in the renormalization matrices gives rise to a radiative tail falling off like $(\log \omega) / \omega$ for large $\omega$. Therefore non-negative moments of the LCDAs are not well defined and have to be considered with an ultraviolett cut-off.[16, 17, 22, 23]

$$
\left\langle\omega^{N}\right\rangle_{ \pm}(\mu)=\int_{0}^{\Lambda_{U V}} d \omega \omega^{N} \phi_{ \pm}(\omega ; \mu)
$$

For $\phi_{-}$it is interesting to examine the limit

$$
\lim _{\Lambda_{U V} \rightarrow \infty} \int_{0}^{\Lambda_{U V}} d \omega \omega^{N} z_{-, 3}^{(1)}\left(\omega, \omega^{\prime}, \xi^{\prime}\right)=0 \quad N=0,1, \quad z_{-, 3}^{(1)}=\frac{1}{2 \varepsilon} \gamma_{-, 3}^{(1)},
$$

which is relevant for the calculation of the three-particle contributions to the moments:

$$
\begin{aligned}
\int_{0}^{\Lambda_{U V}} d \omega \omega^{N} \phi_{-}(\omega ; \mu)= & 1+\frac{\alpha_{s}}{4 \pi}\left(\int d \omega^{\prime} \phi_{-}\left(\omega^{\prime}\right) \int_{0}^{\Lambda_{U V}} d \omega \omega^{N} z_{-}^{(1)}\left(\omega, \omega^{\prime} ; \mu\right)\right. \\
& \left.-\int d \omega^{\prime} d \xi^{\prime}(2-D)\left[\Psi_{A}-\Psi_{V}\right]\left(\omega^{\prime}, \xi^{\prime}\right) \int_{0}^{\Lambda_{U V}} d \omega \omega^{N} z_{-, 3}^{(1)}\left(\omega, \omega^{\prime}, \xi^{\prime}\right)\right)
\end{aligned}
$$

Therefore as stated in [17] three-particle distribution amplitudes give only subleading contribution to the first two moments $(N=0,1)$ and we have explicitly checked that this statement cannot be extended to higher moments $(N \geq 2)$.

The next step consists in using the renormalization properties as a guide to go beyond the existing models derived from a leading-order sum-rule calculation resulting in $\Psi_{A}=\Psi_{V}[20]$ and to analyze their influence on $\phi_{-}$. Finally, for practical calculations involving three-particle contributions, one would need the evolution kernels for the all relevant LCDAs, which will be the subject of a future work.

\section{Acknowledgments}

Work supported in part by EU Contract No. MRTN-CT-2006-035482, "FLAVIAnet" and by the ANR contract "DIAM" ANR-07-JCJC-0031. 


\section{References}

[1] M. Beneke, G. Buchalla, M. Neubert and C. T. Sachrajda, Nucl. Phys. B 591 (2000) 313 [arXiv:hep-ph/0006124].

[2] M. Beneke, G. Buchalla, M. Neubert and C. T. Sachrajda, Nucl. Phys. B 606 (2001) 245 [arXiv:hep-ph/0104110].

[3] M. Beneke and M. Neubert, Nucl. Phys. B 675 (2003) 333 [arXiv:hep-ph/0308039].

[4] A. G. Grozin and M. Neubert, Phys. Rev. D 55, 272 (1997) [arXiv:hep-ph/9607366].

[5] M. Beneke and T. Feldmann, Nucl. Phys. B 685, 249 (2004) [arXiv:hep-ph/0311335].

[6] S. Descotes-Genon and C. T. Sachrajda, Nucl. Phys. B 650, 356 (2003) [arXiv:hep-ph/0209216].

[7] S. Descotes-Genon and C. T. Sachrajda, Phys. Lett. B 557, 213 (2003) [arXiv:hep-ph/0212162].

[8] S. Descotes-Genon and C. T. Sachrajda, Nucl. Phys. B 693, 103 (2004) [arXiv:hep-ph/0403277].

[9] M. Beneke, T. Feldmann and D. Seidel, Nucl. Phys. B 612, 25 (2001) [arXiv:hep-ph/0106067].

[10] M. Beneke and T. Feldmann, Nucl. Phys. B 592, 3 (2001) [arXiv:hep-ph/0008255].

[11] G. Bell, arXiv:0902.1915 [hep-ph].

[12] G. Bell, Nucl. Phys. B 795 (2008) 1 [arXiv:0705.3127 [hep-ph]].

[13] M. Beneke and S. Jager, Nucl. Phys. B 768 (2007) 51 [arXiv:hep-ph/0610322].

[14] M. Beneke and S. Jager, Nucl. Phys. B 751 (2006) 160 [arXiv:hep-ph/0512351].

[15] V. Pilipp, Nucl. Phys. B 794 (2008) 154 [arXiv:0709.3214 [hep-ph]].

[16] B. O. Lange and M. Neubert, Phys. Rev. Lett. 91 (2003) 102001 [arXiv:hep-ph/0303082].

[17] G. Bell and T. Feldmann, JHEP 0804 (2008) 061 [arXiv:0802.2221 [hep-ph]].

[18] V. M. Braun, D. Y. Ivanov and G. P. Korchemsky, Phys. Rev. D 69, 034014 (2004) [arXiv:hep-ph/0309330].

[19] A. Khodjamirian, T. Mannel and N. Offen, Phys. Lett. B 620 (2005) 52 [arXiv:hep-ph/0504091].

[20] A. Khodjamirian, T. Mannel and N. Offen, Phys. Rev. D 75 (2007) 054013 [arXiv:hep-ph/0611193].

[21] H. Kawamura, J. Kodaira, C. F. Qiao and K. Tanaka, Phys. Lett. B 523 (2001) 111 [Erratum-ibid. B 536 (2002) 344] [arXiv:hep-ph/0109181].

[22] S. J. Lee and M. Neubert, Phys. Rev. D 72 (2005) 094028 [arXiv:hep-ph/0509350].

[23] H. Kawamura and K. Tanaka, arXiv:0810.5628 [hep-ph].

[24] S. Descotes-Genon and N. Offen, arXiv:0903.0790 [hep-ph].

[25] B. Geyer and O. Witzel, Phys. Rev. D 72 (2005) 034023 [arXiv:hep-ph/0502239].

[26] G. P. Korchemsky and A. V. Radyushkin, Nucl. Phys. B 283 (1987) 342.

[27] I. A. Korchemskaya and G. P. Korchemsky, Phys. Lett. B 287 (1992) 169.

[28] I. I. Balitsky and V. M. Braun, Nucl. Phys. B 311 (1989) 541.

[29] A. P. Bukhvostov, G. V. Frolov, L. N. Lipatov and E. A. Kuraev, Nucl. Phys. B 258 (1985) 601. 